\title{
El desarrollo y beneficio de las escuelas de odontología sustentables.
}

\section{The development and benefit of sustainable dentistry school.}

\author{
Jesús Rivas Gutiérrez,* María del Carmen Gracia Cortés ${ }^{\ddagger}$
}

\section{RESUMEN}

Las Escuelas de Odontología como espacios educativos universitarios son un lugar idóneo para trabajar de manera urgente los procesos de ambientalización curricular que permitan realizar de forma horizontal y vertical temáticas y contenidos para crear y recrear un tipo de cultura y conciencia más amigable con el medio ambiente, y contribuir con conductas y actitudes sustentables en los estudiantes, docentes y trabajadores administrativos hacia una reducción significativa en la contaminación del ambiente local, regional y mundial, colaborando en ello con la solución a la regularización del clima y el control de los desastres ocasionados por el cambio climático. Además de esto, a ser las Escuelas de Odontología instituciones educativas que pueden funcionar bajo el paradigma de la sustentabilidad, se lograría contribuir al aseguramiento de los recursos naturales renovables y no renovables para las nuevas generaciones, además del logro de un importante ahorro económico en el gasto corriente de las escuelas.

Palabras clave: Desarrollo sustentable, ambientalización curricular, odontología sustentable.

\section{ABSTRACT}

The Dental School as university educational spaces are an ideal place to urgently work on the curricular greening processes that allow working horizontally and vertically with themes and contents that allow creating and recreating a type of culture and awareness that is more friendly to the environment and contribute with sustainable behaviors and attitudes in students, teachers and administrative workers a more significant reduction in pollution of the local, regional and global environment, collaborating in this with the solution to the regularization of the climate and the control of disasters caused by change climate. In addition, being the School of Dentistry educational institutions that can function under the paradigm of sustainability, it would be possible to contribute to the assurance of renewable and non-renewable natural resource for the new generations, in addition to achieving significant economic savings in current spending from school.

Keywords: Sustainable development, curricular greening, sustainable dentistry.

\section{INTRODUCCIÓN}

$E^{n}$ n estos tiempos tan difíciles y complicados social, económica y ambientalmente, las Escuelas de Odontología son consideradas como estructuras organizadas y complejas, con una estrecha relación entre su ideología, organización e infraestructura física, partes que se integran y trabajan por medio de las funciones sustantivas para lograr el fin educativo definido que es la adquisición de las competencias necesarias y requeridas para que al egresar los recursos humanos formados se inserten al ámbito laboral y su entorno social-profesional y coadyuven en la solución de muchos de los problemas que las sociedades tienen. Estas instituciones educativas odontológicas funcionan y trabajan bajo la perspectiva de lograr una adecuada y significativa proyección que permita a los egresados contribuir con su trabajo al desarrollo local, regional y nacional. En ese propósito, y con la educación que se imparte en ellas, se busca que este proceso formativo sea el medio para lograr los fines

\footnotetext{
* Unidad Académica de Odontología, Universidad Autónoma de Zacatecas (UAZ). Zacatecas, México.. Unidad Académica de Docencia Superior, UAZ.

‡ Unidad Académica de Odontología, UAZ.

Recibido: 28 de abril de 2021. Aceptado: 18 de mayo de 2021.

Citar como: Rivas GJ, Gracia CMC. El desarrollo y beneficio de las escuelas de odontología sustentables. Rev ADM. 2021; 78 (4): 209-214. https:// dx.doi.org/10.35366/101075
} 
que curricularmente se pretenden y que los estudiantes y egresados identifiquen las oportunidades que se les presentan para su desarrollo.

Estas funciones sustantivas que se realizan dentro de estas escuelas son el reflejo de la evolución y transición que la educación universitaria ha tenido a lo largo del tiempo. La educación universitaria surge en el S. XIII en París, Italia e Inglaterra, dónde la única función era instruir y adoctrinar; ${ }^{1}$ con el paso de los años y la inclusión de diferentes formas de pensamiento, llegó el resurgimiento de la educación superior moderna, la cual crece en el año de 1810 con la creación de la Universidad de Berlín en Alemania con una nueva visión para la docencia e investigación, después la Universidad de Cambridge en Inglaterra desarrolló y aplicó funciones y acciones como elemento de enlace dinámico entre la Universidad y la sociedad y lo denominó extensión universitaria, iniciando así su modernización y ampliando la vinculación y los beneficios formativos de la cultura e investigación a los diversos sectores poblacionales. ${ }^{2}$

En la antigüedad, la educación universitaria y su proceso educativo estuvo durante mucho tiempo enclaustrada a los límites físicos que formaban el espacio universitario, pero gracias en gran parte al surgimiento de la tercera función sustantiva llamada extensión, se permitió a los universitarios salir de los espacios físicos institucionales y proyectarse socialmente en sus comunidades de forma activa. Morales y colaboradores ${ }^{2}$ han planteado que estas tres funciones en interacción permitieron responder a los diversos contextos históricos cambiantes y funcionaron como detonantes de la democratización de la enseñanza, incluso influir en los procesos de desarrollo político y económico de los países y estados. De manera paulatina, sus enfoques se fueron encauzando en tres intenciones básicas, el más antiguo y más amplio de corte cultural consistió en la transmisión de saberes, conocimientos, habilidades, aptitudes, valores y experiencias que se interiorizaban en los estudiantes, y que con el paso del tiempo cimentaron un tipo de cultura específica; una segunda orientación se basó en la intención de ponderar un enfoque social en el aprendizaje, el cual tenía la finalidad de lograr que los estudiantes, al egresar, se preocuparan por identificar, conocer, comprender y buscar soluciones a los problemas de la sociedad. ${ }^{3}$ El tercer rumbo y más reciente se construyó a partir de pensar a la Universidad como una empresa, siendo esta filosofía aplicada y desarrollada implícita o explícitamente en la mayoría de los países desarrollados de Europa Occidental y Norteamérica, donde permeaba una política económica neoliberal que influyó para que estas instituciones asumieran como directriz educativa el desarrollo industrial del país y se viera a la educación superior como la prestadora de un servicio remunerativo y competitivo.

Aun cuando se puede evidenciar que muchas Universidades del mundo desarrollan actividades tratando de englobar los tres enfoques (cultural, social y empresarial), es evidente que en la mayoría de los casos sobresale el tercero, permeado por los factores del entorno local, regional, nacional y mundial. Desafortunadamente, por sí mismo este enfoque ha llevado a las Universidades y a las Escuelas de Odontología, como parte de la amplia gama de educación universitaria, a la creación y desarrollo de ámbitos y mercados paralelos a él como consecuencia de ello para satisfacer la demanda de insumos para el cumplimiento de su función y misión; uno de estos ámbitos, que se han ido desarrollando constantemente y a paso acelerado en estas escuelas en particular, es el uso y desarrollo de la tecnología y el consumo de desechables dentro de los diversos y amplios procesos formativos que se construyen, crean y recrean en sus aulas, laboratorios y principalmente en las clínicas, sobre todo en lo que respecta a la hora de la realización de los tratamientos y procedimientos clínico odontológicos; esta situación, de manera directa o indirecta, ha contribuido en gran parte al statu quo que guarda el medio ambiente en el ámbito local, regional y mundial, situación conocida como crisis ambiental y originada por las acciones antropocéntricas emprendidas de forma descontrolada y sin responsabilidad social por docentes y alumnos, muchas veces sin planeación y que se manifiestan en la creciente contaminación generada por los desechos excesivos, producto de estas actividades. Este tipo de situaciones de docencia oculta y beneficios inmediatos pero con consecuencias colaterales de contaminación generan una cultura de consumo compulsiva de bienes diversos entre los docentes, estudiantes, trabajadores administrativos y egresados que, en lugar de reducir la carga contaminante en el ambiente, la incrementan.

En la actualidad, como consecuencia de la situación tan crítica del uso y abuso de los recursos no renovables, y de la voraz y excesiva utilización y generación de productos desechables contaminantes y no degradables que se han estado produciendo con las prácticas formativas y profesionales en las Escuelas de Odontología a nivel mundial, ha existido desde hace ya tiempo un desequilibrio climático, reducción en la producción de alimentos, incendios descontrolados, inundaciones atípicas, migración de flora y fauna, aparición de nuevas enfermedades y otras cosas más; por ello, la Organización de las Naciones 
Unidas (ONU), a través de la Organización de las Naciones Unidas para la Educación, la Ciencia y la Cultura (UNESCO), ha trabajo intensamente en establecer nuevas políticas educativas que implican reestructurar los currículos para implementar nuevas finalidades u orientaciones curriculares verticales y/o horizontales para lograr generar como parte de la formación especializada o integral de los alumnos una cultura que les permita ser parte de las acciones para detener, con educación más amigable y pro ambiental, el deterioro ambiental y paralelamente detonar el desarrollo sustentable, empezando por la escuela misma y siguiendo con la comunidad, la región y el país para evitar con ello que siga creciendo la llamada crisis ambiental.

En todo el mundo, como parte de la jerga social y académica, se maneja el término «crisis ambiental» para señalar el estado que guarda el medio ambiental natural y artificial como consecuencia de la gran contaminación que hay; para entender este concepto, partimos de una definición base planteada por Enrique Leff, ${ }^{4}$ quien la precisa como la irracionalidad y pérdida de respeto hacia lo ambiental debido a los modelos arbitrarios de producción y consumo que marca los límites del crecimiento económico de un país o región, dicha crisis viene a cuestionar la racionalidad y los paradigmas teóricos que han impulsado y legitimado el crecimiento económico, negando la importancia de la naturaleza.

La crisis ambiental descontrolada que tenemos hoy está ejerciendo una amenaza tal sobre el planeta que ya no puede darse por seguro que los ecosistemas de la Tierra logren mantener su capacidad para sostener a las generaciones actuales sin poner en riesgo a las futuras. ${ }^{5}$ Hernández y colaboradores ${ }^{6}$ en los análisis ambientales que hicieron en su tiempo, con una visión futurista y realista, manifestaron que la crisis ambiental provocada por el impacto de las actividades humanas y el modelo de vida occidental se unirían a otros síntomas desestabilizadores, como la alteración y desequilibrio económico en muchos países con fuertes desigualdades mundiales en las condiciones de vida de sus habitantes, esta situación ha estado generando en las sociedades humanas actividades que están superando las capacidades de regeneración del planeta, pues ya es muy difícil mantener las infraestructuras, estilos de vida, alimentación y formas de transporte de cada sociedad al sobrepasar la producción natural de recursos naturales que puede otorgar la biosfera, por lo que nos estamos gastando estos recursos más rápido de lo que se pueden producir y renovar, aunado a la dificultad planetaria de absorción de residuos tóxicos, en definitiva esto está modificando la disponibilidad de recursos no renovables y renovables llevando al colapso a los ecosistemas y a las sociedades humanas.

Es claro que la sociedad, influenciada por la globalización, la mercadotecnia y el consumismo desmedido, ha generado bastantes hábitos inconscientes con tal de satisfacer necesidades que ha inventado; según la UNESCO, son tres explicaciones posibles respecto a la necesidad de satisfacerlas: la sobrepoblación, los estilos de vida modernos y el comportamiento individual. Entre otras cosas, estos fueron los motivos que no sólo dieron paso a la crisis ambiental, sino que se sigue agravando, por lo que la ONU ha declarado que «se requieren medidas urgentes ahora». ${ }^{7}$ Es así como surge la búsqueda para confrontar la crisis ambiental global, siendo una alternativa urgente la procuración de una educación superior con orientación hacia el desarrollo sustentable a través de la ambientalización de los currículos como una de las maneras más factibles y pertinentes para enfrentarla, tarea bastante compleja, difícil y ardua en estos nuevos tiempos de incertidumbre laboral, social y ambiental ocasionado por la complejidad de su abordaje debido a la expansión de los cambios discontinuos que provocan rupturas paradigmáticas en las instituciones y organizaciones, a la interdependencia y competitividad de las mismas y a la complicación para establecer las dimensiones, factores y variables ambientales locales y globales para su abordaje. Laurence y Lorsch (loc. cit Lamattina y Donato) ${ }^{8}$ determinaron que en la actualidad la incertidumbre del medio ambiente se debe a la falta de claridad de la información relacionada con el desarrollo de acontecimientos que ocurren en diversos sectores ambientales, a la falta de claridad en las relaciones causa-efecto y a sus repercusiones en las instituciones sociales, económicas y educativas, todas estas situaciones, que deben de ser consideradas a la hora de planear un proceso educativo ambientalizado y continuamente actualizado, hacen la tarea más extrema pero no imposible, laboriosa pero factible.

\section{AMBIENTALIZACIÓN CURRICULAR}

De manera específica, es un proceso reflexivo y de acción orientado a integrar principalmente de manera horizontal a la educación ambiental en el currículo, este proceso debe permitir al docente y estudiante el análisis del contexto socioambiental y la búsqueda de alternativas coherentes con valores de sustentabilidad. Además, conlleva trabajar y adquirir competencias de pensamiento complejo y global con relación al medio, para así fomentar la responsabilidad, el compromiso y la acción de la comunidad educativa hacia el desarrollo de su identidad 
ambiental. ${ }^{9}$ La ambientalización curricular es también un proceso continuo de producción cultural, que tiende a la formación de profesionales comprometidos con la búsqueda permanente de las mejores relaciones posibles entre la sociedad y la naturaleza, aplicando principios éticos y el respeto a las biodiversidades. ${ }^{10}$

De esta manera, dicho proceso implica la introducción de contenidos y criterios ambientales y sustentables en los planes de estudio para proporcionar a los estudiantes conocimientos, habilidades, actitudes y valores que les permitan desarrollarse de manera profesional con respecto al medio ambiente, dentro de los esquemas de un desarrollo sustentable que permita comprender y apreciar el medio ambiente, su complejidad, la relación entre la actividad humana e integrar el factor ambiental en su actividad profesional. Por último, el reto de la ambientalización consiste en incorporar al currículo el conocimiento gestado en el nuevo paradigma del desarrollo sustentable, basándose en saberes interrelacionados que sean capaces de descubrir y entender a la Tierra como sistema y comprender las múltiples relaciones del hombre con la naturaleza, atendiendo y valorando las emociones, las creencias y las capacidades de las personas. ${ }^{11}$

Si las Escuelas de Odontología toman esta postura como referente, deben pensar educar bajo la visión paradigmática del desarrollo sustentable, y repensando las tres dimensiones angulares del mismo desarrollo, las cuales son interdependientes: economía, medio ambiente y sociedad. Esta trilogía busca un crecimiento educativo a largo plazo sin dañar el medio ambiente y los ecosistemas, sin consumir sus recursos de forma indiscriminada, además de lograr un desarrollo equilibrado haciendo un uso eficiente de los recursos naturales, renovables y no renovables a través del proceso educativo y cultural impartido en las escuelas que son o quieren ser sustentables. ${ }^{12}$

\section{ESCUELAS DE ODONTOLOGÍA SUSTENTABLES}

Pensando en la educación ambiental como el medio y a las Escuelas de Odontología como el contexto para lograr el desarrollo sustentable como fin, la educación que ahí se imparta debe funcionar dentro de este espacio escolar para la formación y promoción de la sustentabilidad en donde se formen Profesionistas de la Odontología con una estrecha relación de bienestar, basado en la simbiosis entre el ser humano con su medio, abarcando lo ambiental, cultural, social, económico, político y geográfico, además de formar en el estudiante un pensamiento crítico y resolutivo para que sean agentes de cambio en su entorno inmediato y formar ciudadanos responsables en lo profesional, social y ambiental, en donde se incluyan valores, conciencia y cultura ambiental que lo encaminen al desarrollo sustentable.

En la World Wide Web o red informática mundial (WWW) hay muchos sitios que hacen referencia a la aplicación de acciones sustentables en las escuela, por ejemplo ArchDaily es un sitio web de arquitectura donde se plantea que hay siete principios fundamentales con los que debe contar una escuela sustentable, con base en su construcción y estructura, las cuales son: utilización de energías renovables, acondicionamiento térmico pasivo, captación de agua de lluvia, reutilización de aguas, consumo de alimentos naturales, utilización de materiales reciclados y por último factor humano; una escuela con estas características necesita ser habitada y cuidada para su óptimo funcionamiento con trabajo en equipo, participación diversa, cuidado del medio ambiente y uso responsable de los recursos. ${ }^{13}$

Del mismo modo, Lazovska ${ }^{14}$ sugiere otras características además de las ya mencionadas anteriormente bajo el paradigma de la sostenibilidad: realizar compras con anticipación, comprar bienes de consumo energéticamente eficientes y sustentables, animar el ciclismo, reducir el desperdicio de alimentos y envases, reutilizar antes de tirar, reciclar, dedicar tiempo para el desarrollo profesional y la reflexión del mundo, promover el optimismo y la acción en el entorno, comprometer a las escuelas con la biodiversidad y lograr que entiendan cómo es que se interconecta toda la vida. Muchas veces creemos que las Escuelas de Odontología son sólo centros educativos para aprender asignaturas y contenidos propios del campo disciplinar, pero se debe buscar que se implementen distintos métodos educativos y formativos para convertirlas en espacios sustentables, dado que una comunidad escolar debe crear un plan integral para brindar un impacto positivo en la sociedad, la economía y el medio ambiente, además del compromiso y la participación de estudiantes, docentes y padres de familia; ${ }^{15}$ en otras palabras, estas escuelas deben forjar alumnos que, además de sus conocimientos disciplinares, tengan conocimientos ambientales y reglas de conducta basadas en el reciclaje, conservación, estilo de vida pro ambiental, ahorro de servicios públicos y compras responsables y ecológicas.

Entre otras cosas ya mencionadas, en estas escuelas y dentro de sus espacios clínicos y de laboratorio se debe vigilar y supervisar el manejo adecuado de los RPBI (Residuos Peligrosos Biológico Infecciosos) por personal capacitado para ello, establecer contrato con una empresa profesional para su traslado final e inactivación, dentro de las áreas clínicas y durante la realización de 
los procedimientos médico-odontológicos eliminar los vasos desechables, utilizar batas para cirugía, gorros cubrepelo y cubrezapatos de tela en lugar de desechables, puntas de succión metálicas y reusables, sustituir el uso de amalgamas por resinas, emplear radiografías digitales, utilizar sustancias antisépticas y ecológicas para desinfectar entre paciente y paciente la unidad dental y el equipo fijo como las piezas de mano, descansa brazos y cabezal, lámpara, charolas y todas las superficies que posiblemente fueron salpicadas, o utilización en ellos de barreras de plástico biodegradables no contaminante, evitando el uso de plástico, lavar, desinfectar y esterilizar el instrumental con sustancias biodegradables, en las oficinas descartar el uso de unicel y plástico desechable en vasos, platos y cucharas y sustituirlo por tazas y vasos lavables y reutilizables, elaborar oficios y memorándum de forma digital, eliminando o disminuyendo al mismo tiempo las copias de los mismos, sustituir las lámparas viejas y en serie por lámparas ahorradoras o led con sensor de movimiento en donde sea posible, principalmente en pasillos y patios, sustituir las llaves de agua convencionales por llaves ahorradoras, utilizar en la maquinaria aceites biodegradables, colocar en pasillos y lugares estratégicos botes de basura para separar residuos orgánicos, inorgánicos, pet, aluminio y papel con colores y rótulos diferenciados, contar con una agenda de trabajo preventivo de las unidades dentales y equipo para evitar continuas descomposturas y con ello compra de nuevos equipos con mayor frecuencia que implica generar basura electrónica contaminante y sobre todo realizar periódicamente campañas de concientización ambiental en la comunidad y con pacientes y acompañantes a través de señalética, posters, trípticos, conferencias, talleres, cursos y organización y participación activa de la comunidad en campañas de recolección de basura, reforestación y recuperación de áreas verdes en la escuela y la comunidad.

De esta manera, se construirá culturalmente un tipo de comportamiento específico en todos los miembros de la comunidad odontológica, debido a que los resultados ambientales de toda una sociedad no dependen estrictamente del comportamiento sustentable individual, sino del entrelazamiento en diferentes niveles: escuelas, sectores y grupos, las cuales en conjunto determinan los resultados. Es por ello que, a través de la educación ambiental en estas escuelas orientadas hacia la sustentabilidad, se pueden aprender los distintos patrones de interacción con el medio ambiente natural y artificial.

Una Escuela de Odontología que se plantea educar sobre el desarrollo sustentable es necesariamente una institución que apuesta por ser transformadora, por cambiar estilos de sentir, pensar y actuar, asumiendo la importancia de educar para la acción acompañada de un proceso de reflexión, es también un proyecto educativo que posibilita construir una cultura propia que determina un estilo de organización, toma de decisiones, relación entre sus miembros y dinámicas que constituyen la vía curricular para avanzar hacia una educación para la sustentabilidad. Además, debe ser un proyecto educativo en el que la interacción y la comunicación entre el alumnado, el docente y los demás miembros de la comunidad adquieran un lugar relevante en la toma de decisiones en pro de su medio ambiente y del centro escolar vinculado con su propio entorno. Asimismo, es necesario que la educación que ahí se imparte atraviese las barreras de la Institución para abrirse al medio exterior, en donde se desarrollen estrategias para facilitar que el alumnado sea participe de los proyectos y los problemas del mundo, disponga de herramientas para analizarlos y para entender cuál es su papel y su responsabilidad. ${ }^{16} \mathrm{~A}$ pesar de la creciente incertidumbre ambiental imperante, por su lugar social las Escuelas de Odontología pueden contribuir en una formación deseable de la sociedad completa mediante la construcción de escenarios de sustentabilidad, incluyendo la participación, distribución equitativa, interculturalidad, creatividad, autorrealización, autonomía cultural, siendo estas algunas otras características de las escuelas sustentables. ${ }^{17}$

\section{CONCLUSIONES}

De manera básica, podemos decir que algunos de los beneficios que tiene ser una Escuela de Odontología Sustentable es que protege al ambiente por medio de la generación de una cultura de uso responsable en recursos y servicios ambientales, formando generaciones conscientes del impacto ambiental de sus acciones, también tienen el beneficio de generar ahorros financieros al adoptar medidas eficientes en el uso de servicios, de igual forma se fortalecen los lazos de la comunidad escolar, creando un ambiente óptimo y con relaciones sociales sanas. Asimismo, se enriquece el currículo escolar, abriendo las posibilidades a un panorama más amplio de contenidos, actividades, programas y proyectos; por último, mejora la imagen de la escuela al convertirse en un factor de cambio no sólo en la institución, sino también en la localidad, volviéndose una opción educativa más eficiente, atractiva y de gran impacto positivo para la sociedad, generando en sus estudiantes un aumento de imaginación y creatividad, la obtención de una visión más global que permita apreciar la interconexión entre problemas sociales, ecológicos, económicos, culturales 
y políticos, se potencia el pensamiento crítico que incita a investigar cómo y por qué suceden las cosas para así poder tomar decisiones propias sobre problemas ambientales complejos, también se incrementa la tolerancia y la compresión, se alienta un estilo de vida saludable, se fortalecen las comunidades, en consecuencia se protege al medio ambiente, a los estudiantes, docentes y el resto del personal de la Institución aprende a ser mejor por medio del aprendizaje activo, conocen nuevas técnicas de enseñanza-aprendizaje y se les crea la potencialidad de tener liderazgo estudiantil al permitirles dejar una marca en su escuela y comunidad, todo esto además favorece la imagen de la escuela y la Universidad a la que pertenecen al ofrecer una mejor y más pertinente educación para las actuales $y$ futuras generaciones.

\section{REFERENCIAS}

1. Restrepo P. Origen de las universidades en general y de la Universidad de San Buenaventura en especial. Management. 1999; 8 (11): 15-28.

2. Morales M, Mira G, Arias M. Enfoques y retos de la función de extensión universitaria como mecanismos de integración: Universidad, Empresa, Estado. II Congreso Internacional de Gestión Tecnológica e Innovación realizado en Bogotá D.C., Colombia. 2010.

3. Vega MJF. La extensión universitaria: función integradora del quehacer universitario en el presente siglo. Investigación y Desarrollo. 2002; 10 (1): 26-39.

4. Leff E. Saber ambiental: sustentabilidad, racionalidad, complejidad, poder. Siglo XXI. 2002.

5. Sosa SB, Isaac-Márquez R, Eastmond A, Ayala ME, Arteaga MA. Educación superior y cultura ambiental en el sureste de México. Universidad y Ciencia. 2010; 26 (1): 33-49.

6. Hernández A et al. La crisis ecosocial en clave educativa. Guía didáctica para una nueva cultura de paz. Madrid, España: Centro de Investigación para la Paz (CIP-Ecosocial). 2010.

7. UNESCO. La educación al servicio de los pueblos indígenas: Creación de futuros sustentables para todos. París: Ediciones UNESCO. 2016.

8. De Ferrai ML, Ferrari ND. "Scanning" las dimensiones ambientales: incertidumbre, cambios y complejidad. Revista Científica de la Universidad de Mendoza, Argentina. 2013; 1: 3.
9. Geli AM, Junyent M, Medir R, Padilla F. La ambientalización curricular en la enseñanza obligatoria: una propuesta de definición, caracterización y estrategias, Barcelona: Departamento de Medio Ambiente y Vivienda. 2006.

10. Bravo MMT. La UNAM y sus procesos de ambientalización curricular. Revista mexicana de investigación educativa. 2012; 17 (55): 1126-1134.

11. Agoglia OB, Erice MX. Diagnóstico del grado de ambientalización de la Facultad de Educación Elemental y Especial. Universidad de Cuyo (Argentina). Ambientalización Curricular de los Estudios Superiores. Diagnóstico de la Ambientalización Curricular de los Estudios Superiores. Diversitas. 2003; 46: 21-46.

12. SEMARNAT (Secretaría de Medio Ambiente y Recursos Naturales). Diferencia entre sustentable y sustentable. 2018. [Consultado: 04 de agosto de 2020] Recuperado de URL: https:// www.gob.mx/semarnat/articulos/diferencia-entre-sustentable-ysustentable

13. Arch Daily. 7 Principios en la construcción de una escuela sustentable. 2018. [Consultado el 15 de agosto del 2020] Recuperado de URL: https://www.archdaily.mx/mx/893477/7principios-en-la-construccion-de-una-escuela-sustentable-demichael-reynolds-en-argentina

14. Lazovska D. 16 Tips para hacer más sustentable a una escuela. Expok. 2019; [Consultado el 16 de agosto del 2020] Recuperado de URL: https://www.expoknews.com/16-tips-para-hacer-massustentable -a-una-escuela/

15. López M. Características de una escuela sustentable. 2016. [Consultado el 18 de agosto del 2020] Recuperado de URL: https://www.expoknews.com/caracteristicas-de-una-escuelasustentable/

16. Pujol RM. Construir una escuela que eduque para el desarrollo sustentable. La sostenibilidad, un compromiso de la escuela. Barcelona: Grao. 2006, 21-26.

17. Martínez-Castillo R. La importancia de la educación ambiental ante la problemática ambiental. Revista electrónica educare. 2010; 14 (1): 97-111. [Consultado el 22 de agosto del 2020] doi: https:// doi.org/10.15359/ree.14-1.9.

Correspondencia:

Jesús Rivas Gutiérrez

E-mail: rivasgutierrez@hotmail.com

Conflicto de intereses: Ninguno.

Financiamiento: Ninguno. 